\title{
Management of Liver Trauma in Kuwait
}

\author{
Sami Asfar ${ }^{a-c}$ Mousa Khoursheed ${ }^{b, c}$ Mervat Al-Saleh ${ }^{b, c}$ Abdullah A. Alfawaz $^{a}$ \\ Medhat M. Farghaly ${ }^{d}$ Ali M. Nur e on behalf of the Liver Trauma Registry Group \\ ${ }^{a}$ The Liver Surgery Unit and b ${ }^{b}$ Department of Surgery, Mubarak Al-Kabeer Hospital, ' Department of Surgery, Faculty \\ of Medicine, Kuwait University, ${ }^{\mathrm{d}}$ Department of Surgery, Al-Adan Hospital, and ${ }^{\mathrm{e}}$ Department of Surgery, Al-Jahra \\ Hospital, Kuwait, Kuwait
}

\author{
Key Words \\ Non-operative management of liver trauma $\cdot$ Blunt liver \\ injuries · Damage control · Perihepatic packing · Abdominal \\ compartment syndrome
}

\begin{abstract}
Objectives: The aim of this study was to introduce the concept of non-operative management (NOM) for blunt liver trauma by establishing a protocol and a prospective Liver Trauma Registry in Kuwait. Subjects and Methods: A prospective Liver Trauma Registry was started in 4 hospitals and it included 117 patients who had sustained blunt liver trauma (94 men and 23 women). Unstable patients were taken to surgery while stable patients were managed conservatively regardless of the grade of liver injury. High-grade (III$\mathrm{VI})$ liver injuries were managed in collaboration with the liver surgery specialist. Results: The mean age of the 117 patients was $29.02 \pm 11.18$ years (range 7-63). NOM was successful in 94 (96\%) patients and failed in 4 (4\%) (these 4 then underwent successful surgery). Nineteen (16.2\%) were unstable and underwent surgery immediately; 15 (79\%) of them survived (they had had grade III-V injuries) and 4 died ( 2 with grade $\mathrm{V}$ injuries and 2 with grade $\mathrm{VI}$ injuries). Perihepatic packing was necessary in $8 / 19(42 \%)$ patients. The overall mortality was $3.4 \%$ (4/117). Conclusions: This study showed that NOM was successful in a majority of patients with blunt
\end{abstract}

liver trauma. In addition, it confirmed that the magnitude of liver injury and haemoperitoneum did not preclude NOM as long as the patient was haemodynamically stable.

(c) 2014 S. Karger AG, Basel

\section{Introduction}

Non-operative management (NOM) of blunt liver injuries in haemodynamically stable patients has become the standard therapeutic modality in most trauma centres [1]. Success rates of more than $90 \%$ have been consistently reported [2-4]. Many factors contribute to this success: (1) more precise imaging of the liver with the evolution of computer tomographic (CT) scanning and the advent of multi-detector computed tomography, (2) a better understanding of the liver surgical anatomy and the pathophysiology of liver injury, (3) objective assessment of the severity of liver injury by the universal adoption of the Liver Injury Scale of the American Association for Surgery of Trauma (AAST) [5]. Consistently lower liver-related complication rates $(0-11 \%)$ have been reported with conservative management [6]. Moreover, the recent literature shows that the results of surgery in cases which fail with NOM had likewise improved because of the more generous use of perihepatic packing in high-grade injuries (also used by liver specialists) as opposed to the

Prof. Sami Asfar

Department of Surgery, Faculty of Medicine, Kuwait University PO Box 23924

Safat 13110 (Kuwait)

E-Mail sami@hsc.edu.kw Creative Commons Attribution-NonCommercial 3.0 Unported license (CC BY-NC) (www.karger.com/OA-license), applicable to the online version of the article only. Distribution permitted for non-commercial purposes only. 
earlier enthusiasm for major liver resections or the use of atriocaval shunts $[1,7]$. The success of NOM of blunt liver injuries in the last three decades has encouraged trauma centres to adopt the same policies (though selectively) pertaining to abdominal injuries $[8,9]$.

Kuwait is a small, affluent country with one of the highest rates of road-traffic accidents but it still does not have a level 1 trauma centre. With the establishment of the Liver Surgery Unit in the Mubarak Al-Kabeer Hospital in 2003 , it was decided to introduce NOM protocols. The vision was that if this endeavour produced results equivalent to those from major trauma centres worldwide, it could be a model for countries in this geographical area (all of which have a high incidence of car accidents) to follow. The objective of this study was therefore to introduce the concept of NOM for blunt liver trauma by establishing a protocol for a Liver Trauma Registry in Kuwait.

\section{Subjects and Methods}

Four major hospitals from three regions in Kuwait participated in the registry. Two of them, the Mubarak Al-Kabeer Hospital (the site of the Liver Surgery Unit) and the Sabah Hospital, are in the central region. The Al-Jahra and Al-Adan Hospitals are in the north and south, respectively, situated on the two major highways to the north and south of the country. The surgical units in the participating hospitals strictly followed the protocol for NOM of liver trauma (table 1).

Grading of liver injury was done according to the AAST scale [5] based on contrast-enhanced CT scan of the abdomen. To simplify this grading system, the senior author (S.A.) came up with a simple drawing (fig. 1) which was distributed to all participating surgical units; it enabled non-liver general surgeons to decide the liver injury grade at a glance.

Patients were assessed and managed in their respective hospital. They were managed according to the Advanced Trauma Life Support (ATLS) guidelines. The liver surgeon (S.A.) was available on a 24-hour/7-day basis for all the participating units to oversee the strict implementation of the NOM criteria and he was involved directly or indirectly in all injuries of grade III and higher. Patients requiring surgery were mostly managed in their hospitals by the liver surgeon and the local team. Very few patients required transfer to the Liver Surgery Unit.

A special Liver Trauma Registry form was completed for every patient that including details about liver injury grade (fig. 1), associated injuries, blood transfusion requirements, ICU admission, surgical procedures, complications and ICU and total hospital stay. At the end of care (i.e. at discharge from hospital or death) these forms and CT-scan images were sent to the Liver Surgery Unit, Mubarak Al-Kabeer Hospital, to be included in the information database.

The following protocol was followed in all participating surgical units: (1) haemodynamically unstable patients despite adequate resuscitation underwent emergency laparotomy without any undue delay and (2) haemodynamically stable patients on arrival or after resuscitation were managed according to the NOM guidelines (ta-
Table 1. Guidelines for NOM of liver injuries

Haemodynamically stable patient regardless of the magnitude of liver injury

Absence of peritoneal signs and other abdominal injuries requiring immediate surgery

Good CT scan and grading of liver injury

Replace blood loss from associated injuries e.g. fracture femur, pelvis, haemothorax

Grade I-II injuries can be managed in the ward with close monitoring

Grade III and higher must be managed in the surgical ICU

Blush on initial CT scan

(1) If haemodynamically stable, follow NOM guidelines

(2) Repeat CT scan with i.v. contrast within $24-48 \mathrm{~h}$

(3) Consider percutaneous vascular embolization if repeat CT scan shows persistence of blush

Indications for surgery

(1) Continued need for blood transfusion, exceeding 5 units for liver-related bleeding

(2) Development of peritoneal signs

(3) Unstable vital signs despite resuscitation

(4) Intrahepatic infection

ble 1). It was emphasized that the appearance of the liver on the CT scan or the amount of haemoperitoneum was not an indication for surgery. Injuries that were grade III or higher indicated admission to the surgical ICU with strict bed rest and serial haemoglobin and haematocrit assessment (4- to 6-hourly for the first $24 \mathrm{~h}$ ). After the initial CT scan, all patients had one scan with contrast before discharge from hospital for the purpose of documenting and assessing the condition of the liver. During the hospital stay, further scanning was at the discretion of the treating team for the purpose of assessing the progression of the liver injury, the collection of intraperitoneal or perihepatic fluid or assessing the reasons for continued blood loss.

The presence of contrast extravasation (blush) on the initial CT scan was not an urgent indication for percutaneous vascular embolization. If haemodynamically stable, these patients were treated as NOM patients in the ICU but had a repeat CT scan within 24$48 \mathrm{~h}$. Angioembolization was considered if the haemoglobin and haematocrit levels failed to rise and/or the patient remained haemodynamically unstable or blush persisted on a repeat CT scan.

NOM was discontinued in patients who became haemodynamically unstable, upon discovery of an intrabdominal injury or the development of signs requiring surgical intervention. Upon discharge from hospital, patients were instructed to avoid violent and contact sports for 3 months.

\section{Results}

During the study period (June 2003 to July 2012), 117 patients were managed in the 4 hospitals participating in the Liver Trauma Registry. Of these, 94 (80.3\%) were 
Fig. 1. AAST Liver Organ Injury Scale diagram. Lesions drawn on the left lobe show laceration depth and subcapsular haematoma. Lesions on the right lobe show intraparenchymal haematoma and parenchymal disruption.

Fig. 2. Outcome of management of the 117 patients admitted to the Liver Trauma Registry from 2003 to 2012.
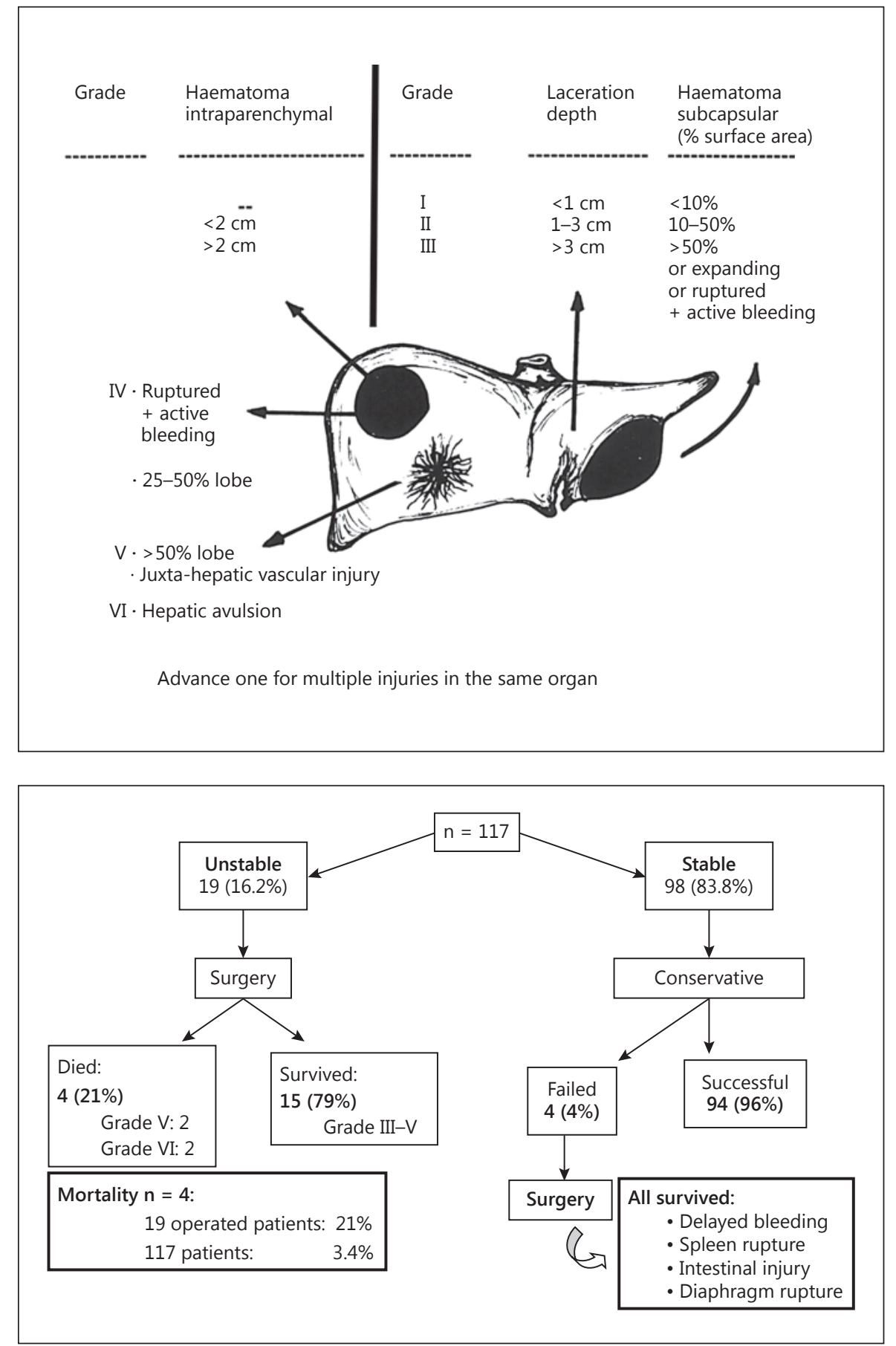

male and $23(19.7 \%)$ were female $(\mathrm{M}: \mathrm{F}=4: 1)$ and the mean age \pm SD was $29.02 \pm 11.18$ years (range $7-63$ ). The average ICU stay was 5.9 days (range $2-10$ ). The mean hospital stay \pm SD was $17.43 \pm 7.95$ days (range 5-67) Ninety-eight $(83.8 \%)$ patients were stable and were therefore managed conservatively (NOM) and 19 (16.2\%) were considered unstable after attempts at resuscitation and so they were taken to surgery (fig. 2).

\section{NOM of Stable Patients}

Of the 98 stable patients, conservative management was successful in $94(96 \%)$ and NOM was discontinued 


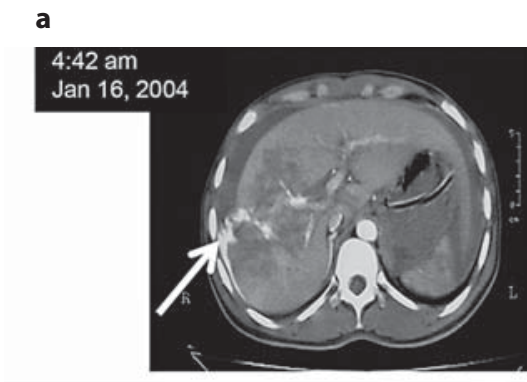

b
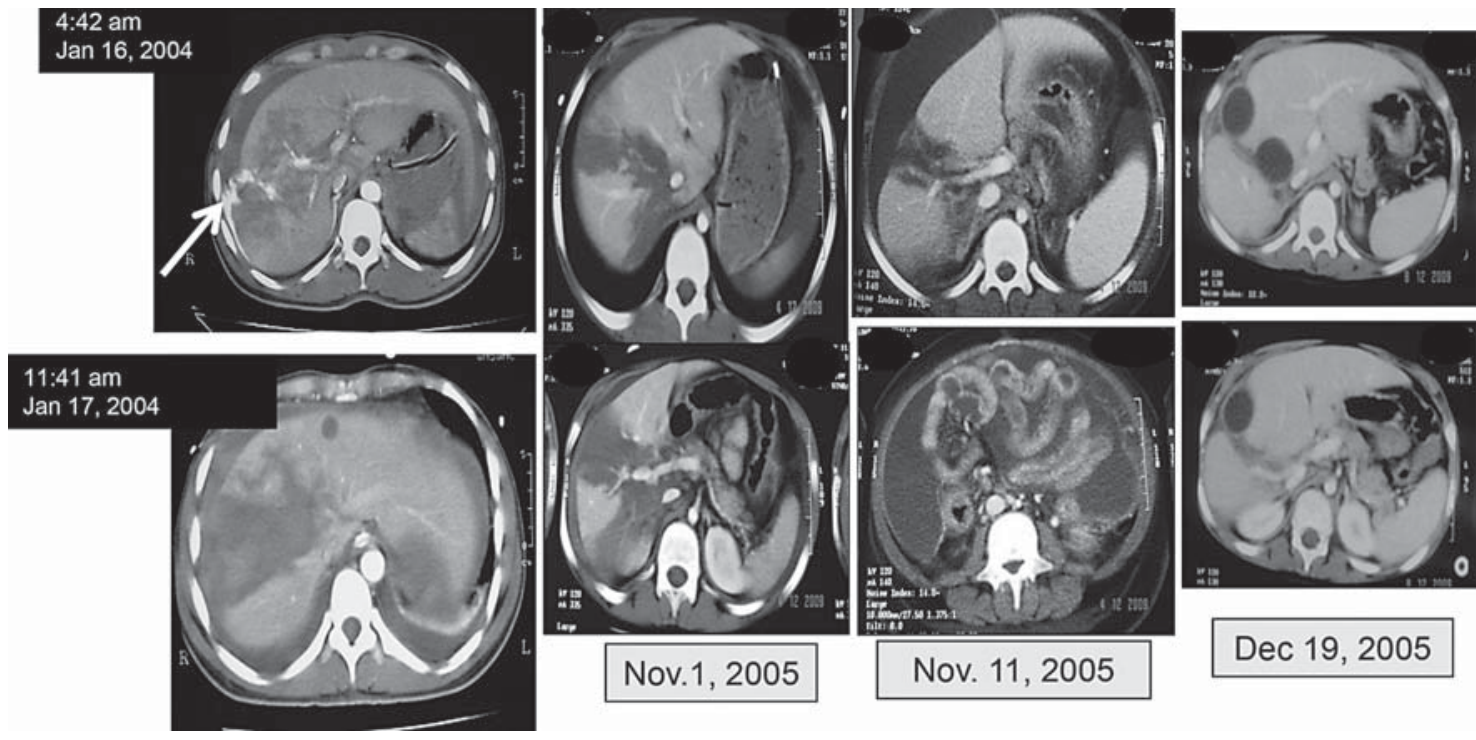

Fig. 3. a A case of blush on CT scan: a 33-year-old man involved in a road-traffic accident. CT scan of the liver at 4:42 a.m. showed a grade IV injury in the right lobe with extravasation of contrast 'blush' (white arrow). He was haemodynamically stable. He was admitted to the ICU and received 1 unit of packed red blood cells. A repeat CT scan at 11:41 a.m. the next day, i.e. $31 \mathrm{~h}$ later, showed no blush and he remained stable with no further blood transfusion required up to discharge from hospital. b A case of abdominal compartment syndrome: an 8-year-old child after a road-traffic accident. November 1, 2005: CT scan of the liver showed a grade $\mathrm{V}$ injury involving segments VIII, VII and V; on arrival, his haemoglobin was $6 \mathrm{~g} / \mathrm{l}$. On November 11 (10th day in ICU), his abdomen became tense with a girth increase from 29 to $32 \mathrm{~cm}$ and signs

of increased intra-abdominal pressure (desaturation, tachycardia $150 /$ min with normal blood pressure and a decrease in urine output). The CT scan showed a large amount of intraperitonial fluid. Under ultrasound guidance, two large-bore percutaneous drains were inserted in the right and left sides of the abdominal cavity. A total of 1,450 $\mathrm{ml}$ of bilious-bloody fluid was drained and the pressure gradually decreased over 1 week. This was followed by a remarkable improvement in his general condition and a normalization of oxygen saturation and pulse rate. Follow-up CT scan on December 5 showed evidence of healing of the liver, no collection of intraperitoneal fluid and only two small residual intrahepatic haematomas.

only in 4 (4\%) due of delayed bleeding (rupture of intrahepatic right-lobe haematoma), splenic rupture, smallbowel injury or rupture of the diaphragm. All these 4 patients survived the surgical procedures (fig. 2).

Seven patients had blush (extravasation of contrast) on the first CT scan of the liver and 4 developed abdominal compartment syndrome during the conservative management. As per the Liver Trauma Registry guidelines (table 1), they were managed non-operatively, i.e. without surgery or vascular embolization, as exemplified in figure 3.

\section{Operative Management of Unstable Patients}

Nineteen unstable patients underwent emergency surgery including intraparenchymal haemostasis, resectional debridement, splenectomy and left liver hepatectomy. Fifteen (79\%) with grade III-V liver injuries survived. Four (21\%) died due to severe liver injuries of grade $\mathrm{V}$ $(\mathrm{n}=2)$ and grade VI $(\mathrm{n}=2) ; 2$ died on table from severe

retrohepatic bleeding and the other 2 died at 9 and $24 \mathrm{~h}$ after surgery from exsanguination and coagulopathy. In addition to their severe liver injuries, these 4 patients had comorbidities (type 2 diabetes mellitus, ischaemic heart disease and hypertension) and they were older than the other patients in the cohort (range 51-63 years). Perihepatic packing was used in 8 (42\%) patients; the packs were removed after $48-72 \mathrm{~h}$ with abdominal closure in all cases. Packing achieved haemostasis in $6 / 8$ patients $(75 \%)$, but 2 patients with grade VI liver injuries died. The overall mortality of the 117 patients in this prospective registry was 4 (3.4\%); all were liver-related deaths (fig. 3).

The grades of the liver injuries of the 117 patients are given in table 2. Grades III, IV and V were the most common $(67.5 \%)$. The blood transfusion requirement was 1-6 units of packed red blood cells. Higher grades (V and VI) required more transfusions ( $>10$ units) and were associated with mortality despite surgical intervention (table 2). 


\section{Complications}

Of the 117 patients, 4 (3.4\%) developed complications. One patient under NOM developed a perihepatic abscess which responded to percutaneous drainage. The other 3 developed bile leaks which were managed by endoscopic retrograde cholangio-pancreatography and stenting. The stents were removed after 6 weeks. One of these bile leaks occurred after 4 weeks of NOM, with the patient suddenly showing signs of shock and with a haemoglobin concentration of $7 \mathrm{~g} / \mathrm{l}$. Resuscitation and laparotomy were performed immediately, and bleeding from a ruptured, right-lobe intrahepatic haematoma was detected. Intraparenchymal haemostasis was satisfactory (Argon beam, haemaclips and haemostatic glue). A drain was left in the subhepatic space which initially drained bloodstained fluid for a few days followed by biliary leak of 300-400 ml per day which continued for about 2 weeks.

\section{Associated Injuries}

Of the 117 patients, $77(65.8 \%)$ had associated injuries that included fractured ribs, lung contusions, haemothorax, fractures, splenic injuries, kidney injuries, diaphragmatic rupture, intestinal injury and adrenal haematoma; 32 of these (41.5\%) had more than one injury. The renal injuries and adrenal haematoma resolved with conservative management. The diaphragmatic and small intestinal injuries were detected upon clinical examination and were confirmed by CT scan; these patients had a successful outcome after surgery. One patient in the non-operative group required a splenectomy due to splenic rupture 1 week after admission. Most patients had some degree of brain concussion, which resolved within their hospital stay, with them regaining full consciousness. None of the patients in this study died from these associated injuries.

\section{Discussion}

Like previous reports, the patients were mostly young males who had sustained road-traffic accidents $[10,11]$. In this study, implementing NOM in haemodynamically stable patients achieved $96 \%$ success and only $4 \%$ failure. These results are consistent with most recent reports on NOM of liver trauma from other centres [ $7,12,13]$. Two main factors contributed to this outcome: first, the set guidelines were strictly implemented by all the participating centres and second, the early involvement of a specialist liver surgeon decreased inter-observer bias which might occur during assessment and management. This
Table 2. Liver injury grades and blood transfusions in our 117 patients

a Grades of liver injury

\begin{tabular}{lll}
\hline Grade & Patients, $\mathrm{n}$ & Patients, $\%$ \\
\hline I & 15 & 12.8 \\
II & 21 & 18 \\
III & 42 & 36 \\
IV & 26 & 22 \\
V & 11 & 9.4 \\
VI & 02 & 1.8 \\
\hline
\end{tabular}

Grade III-V ( $\mathrm{n}=79)$ or $67.5 \%$.

b Blood transfusions

\begin{tabular}{llc}
\hline Blood units, $\mathrm{n}$ & Liver injury grade & Patients, $\mathrm{n}$ \\
\hline 0 & I + II & $36(30.7 \%)$ \\
$1-2$ & III $(\mathrm{n}=34)+\mathrm{IV}(\mathrm{n}=4)$ & $38(32.5 \%)$ \\
$3-6$ & III $(\mathrm{n}=8)+\mathrm{IV}(\mathrm{n}=18)$ & $26(22.2 \%)$ \\
$7-10$ & IV $(\mathrm{n}=4)+\mathrm{V}(\mathrm{n}=6)$ & $10(8.6 \%)$ \\
$>10$ & V $(\mathrm{n}=5)+\mathrm{VI}(\mathrm{n}=2)$ & $7(6 \%)$ \\
\hline
\end{tabular}

policy has shown its effectiveness in optimizing the care of these patients [14].

From our study, it became apparent that the magnitude of liver injury seen on CT scan cannot be used as a criterion to determine the need for surgical intervention. We showed that even higher grades of liver injury responded to NOM and that only a loss of haemodynamic stability or the development of complications determined the need for surgery as previously reported [3, 10-13]. Likewise, the volume of haemoperitoneum did not influence our decision to continue NOM as long as the patient was haemodynamically stable and the blood transfusion requirement for liver-related injuries did not exceed 5 units $[7,9,11,15,16]$. NOM failed in only 4 patients (4\%) due to associated injuries. In comparison, Velmahos et al. [4] reported a failure of $17 \%$. This was perhaps because the number of liver injuries in their report was small, comprising only 72 out of the 206 solid-organ injuries.

In patients undergoing NOM, there is always a concern about missing hollow viscus injuries. In a multi-institutional analysis of 275,557 trauma admissions, Watts et al. [17] found missed hollow viscus injury to be only $0.3 \%$. In our NOM group, there was one intestinal injury and one diaphragmatic rupture (1.0\%) which were discovered early and successfully managed. 
Four (4\%) patients in the NOM group developed increased abdominal compartment pressure (all grade $\mathrm{V}$ liver injuries). The general surgery literature advocates immediate surgery to decompress the abdominal cavity and prevent multi-organ failure; however, it seems that indiscriminately applying this principle to severe liver injuries would have increased morbidity and mortality in this patient cohort $[1,13]$. In our patients who showed early signs of increased abdominal compartment pressure, we opted to 'vent' the peritoneal cavity by two or more wide-bore percutaneous drains, rather than performing immediate laparotomy. This policy was successful in our 4 patients, with none requiring surgery and all showed a steady recovery (fig. 3b). Performing laparotomy in this group with high-grade liver injury would invariably have led to major liver resections which are associated with $50-87 \%$ mortality in this setting $[18,19]$. Though our numbers were small, we see no harm in first considering abdominal cavity venting as soon as a patient starts developing unexplained tachycardia and oxygen desaturation together with an intra-abdominal pressure of grade III (16-25 mm Hg) and/or oliguria, as reported previously [20]. Laparotomy becomes mandatory if peritoneal drainage 'venting' fails to reduce intra-abdominal pressure after $24-48 \mathrm{~h}$.

Many studies recommend that patients with contrast extravasation 'blush' on the initial CT scan should undergo immediate percutaneous embolization (angioembolization) or laparotomy $[4,13,16]$. Though angioembolization can contribute to the success of NOM in liver trauma management, it is not completely innocuous, as it has caused major complications like hepatic necrosis, biloma formation, bile leaks, gall bladder gangrene and intrahepatic abscess formation [6, 21-23]. In a retrospective study by Yuan et al. [24], it was shown that attempts at angioembolization were negative in $26.4 \%(48 / 182)$ of patients who were taken to the angiography suite because of a 'blush' seen on the initial CT scan. Again, we see no harm in trying a watchful policy and a selective use of angioembolization in order to avoid the associated morbidity that can occur, especially in patients with a high-grade liver injury.

The overall mortality in our study was $3.4 \%(4 / 117)$ due to high-grade liver injuries. This result is similar to the $4.9 \%(9 / 183)$ reported by Schnüriger et al. [10]. Even in the hands of specialist liver surgeons, the mortality rate from surgery in this setting was 35\% in the elderly and up to $20 \%$ in the young patients [25]. It was shown that liver resection under emergency conditions in patients with liver injuries greater than grade III was associated with mortality rates in excess of 50\% [26]. In all of our operated patients, we aimed to achieve reasonable haemostasis and tried to avoid major resectional procedures and prolonged surgery. In 2010, Ramkumar et al. [14] reported that the mortality rate of operated patients decreased from 50 to $27 \%$ when hepatic surgeons were involved.

When not able to achieve the minimum haemostasis, we reverted to perihepatic packing before the patient drifted into the 'triangle of death' of hypothermia, acidosis and coagulopathy. We kept continuous communication with the anaesthetist in charge during these procedures, and packing became mandatory when we approached the limit of 10 units of blood transfusion, the arterial $\mathrm{pH}$ started to drop to about 7.2 and the core body temperature decreased to $\leq 34^{\circ} \mathrm{C}$ [1]. Our mortality rate following perihepatic packing was $25 \%$ (2/8); this is lower than the $43 \%(9 / 21)$ mortality reported recently by Leppäniemi et al. [7], perhaps because of the small number of our patients. Ramkumar et al. [14] reported packing in $84 \%(31 / 37)$ of patients requiring surgical management, with haemostasis being achieved in $77 \%$, which is similar to our results.

Recent literature advocates damage control with early perihepatic packing even in the presence of juxtahepatic major venous injury $[1,7,14]$. This policy has stood the test of time and was shown to be effective in $59-77 \%$ of operated liver trauma patients because the hepatic venous system is a low-pressure system and compression would suffice to stop bleeding $[7,14]$. The era of using atriocaval shunting for juxtahepatic bleeding is gone, as it was associated with $80-100 \%$ mortality [26-28] as opposed to the up to $77 \%$ survival achieved by perihepatic packing as shown in our report and that of others $[1,7,14,29]$. Furthermore, perihepatic packing has become a salvage procedure even during elective liver surgery [30].

A major limitation of this study is that it does not represent the full spectrum of liver trauma in the country as 3 other general hospitals did not participate in this registry.

\section{Conclusion}

This study showed that implementing a strategy of NOM for blunt liver injuries and collaboration with a liver surgery specialist in a developing country achieved results similar to those reported from major level 1 trauma centres and saved many young adult lives. Moreover, it confirmed that the magnitude of liver injury and haemoperitoneum does not preclude NOM as long as the patient is haemodynamically stable. 


\section{Acknowledgement}

The authors acknowledge the valuable contributions of The Liver Registry Group in the different hospitals: Emad Ayyash, Ahmad Al-Mosawi, Osama M. Loutfy and Ali Adahham at the Mubarak Al-Kabeer Hospital (Liver Surgery Unit), Nael M. Salman at Al-Adan Hospital, Syed F.H. Belgrami, Bakri H. Tanoum and Farhan M. Alanzi at the Al-Jahra Hospital and Waleed Almasri at the Sabah Hospital. The valuable input and unlimited assistance provided by the medical and nursing staff in all surgical ICUs in the participating hospitals is highly appreciated.

\section{References}

$>1$ Richardson JD, Franklin GA, Lukan JK, et al: Evolution in the management of hepatic trauma: a 25-year perspective. Ann Surg 2000;232: 324-330.

-2 Kozar RA, Moore FA, Moore EE, et al: Western Trauma Association critical decisions in trauma: nonoperative management of adult blunt hepatic trauma. J Trauma 2009;67: 1144-1149.

$\checkmark 3$ Malhotra AK, Fabian TC, Croce MA, et al: Blunt hepatic injury: a paradigm shift from operative to nonoperative management in the 1990s. Ann Surg 2000;231:804-813.

-4 Velmahos GC, Toutouzas KG, Radin R, et al: Nonoperative treatment of blunt injury to solid abdominal organs. A prospective study. Arch Surg 2003;138:844-851.

$\checkmark 5$ Moore EE, Cogbill TH, Jurkovich GJ, et al: Organ injury scaling: spleen and liver (1994 revision). J Trauma 1995;38:323-324.

6 Kozar RA, Moore JB, Niles SE, et al: Complications of nonoperative management of highgrade hepatic injuries. J Trauma 2005;59: 1066-1071.

$>7$ Leppäniemi AK, Mentula PJ, Streng MH, et al: Severe hepatic trauma: nonoperative management, definitive repair, or damage control surgery? World J Surg 2011;35:2643-2649.

-8 Butt MU, Zacharias N, Velmahos GC: Penetrating abdominal injuries: management controversies. Scand J Trauma Resusc Emerg Med 2009; 17:19.

-9 Zafar SN, Rushing A, Haut ER, et al: Outcome of selective non-operative management of abdominal injuries from the North American National Trauma Database. Br J Surg 2011; 99(suppl 1):155-165.

10 Schnüriger B, Inderbitzin D, Schafer M, et al: Concomitant injuries are an important determinant of outcome of high-grade blunt hepatic trauma. Br J Surg 2009;96:104-110.
11 Scollay JM, Beard D, Smith R, et al: Eleven years of liver trauma: the Scottish experience. World J Surg 2005;29:744-749.

12 Parks N, Davis J, Forman D, et al: Observation for nonoperative management of blunt liver injuries: how long is long enough? J Trauma 2011;70:626-629.

13 Clemente N, Di Saverio S, Giorgini E, et al: Management and outcome of 308 cases of liver trauma in a Bologna trauma center in 10 years. Ann Ital Chir 2011;82:351-359.

14 Ramkumar K, Perera MT, Marudanayagam $\mathrm{R}$, et al: A reaudit of specialist managed liver trauma after establishment of regional referral and management guidelines. J Trauma 2010;68:84-89.

15 Shapiro MB, Nance ML, Schiller HJ, et al: Nonoperative management of solid abdominal organ injuries from blunt trauma: impact of neurologic impairment. Am Surg 2001;67: 793-796.

16 van der Vlies $\mathrm{CH}$, Olthof DC, Gaakeer M, et al: Changing pattern in diagnostic strategies and the treatment of blunt injury to solid abdominal organs. Int J Emerg Med 2011; 4:47

17 Watts DD, Fakhry SM; EAST Multi-Institutional Hollow Viscus Injury Research Group: Incidence of hollow viscus injury in blunt trauma: an analysis from 275,557 trauma ad missions from the East Multi-Institutional Trial. J Trauma 2003;54:289-294.

18 Ochai T, Igari K, Yagi M, et al: Treatment strategy for blunt hepatic trauma: analysis of 183 consecutive cases. Hepatogastroenterology 2011;58:1312-1315.

19 Asensio JA, Roldán G, Petrone P, et al: Operative management and outcomes in 103 AAST-OIS grades IV and V complex hepatic injuries: trauma surgeons still need to operate but angioembolization helps. J Trauma 2003; 54:647-653.

-20 Moore AFK, Hargest R, Martin M, et al: Intraabdominal hypertension and abdominal compartment syndrome. Br J Surg 2004;91: 1102-1110.
21 Dabbs DN, Stein DM, Scalea TM: Major hepatic necrosis: a common complication after angioembolization for treatment of highgrade liver injuries. J Trauma 2009;66:621627.

22 Letoublon C, Morra I, Chen Y, et al: Hepatic arterial embolization in the management of blunt hepatic trauma: indications and complications. J Trauma 2011;70:1032-1037.

23 Di Saverio S, Moore EE, Tugnoli G, et al: Nonoperative management of liver and spleen traumatic injuries: a giant with clay feet. World J Emerg Surg 2012;7:3.

24 Yuan KC, Wong YC, Lin BC, et al: Negative catheter angiography after vascular contrast extravasations on computed tomography in blunt torso trauma: an experience review of a clinical dilemma. Scand J Trauma 2012;20: 46-53.

25 Tsugawa K, Koyanagi N, Hashizume M, et al: Anatomic resection for severe blunt liver trauma in 100 patients: significant differences between young and elderly. World J Surg 2002; 26:544-549.

26 Cogbill TH, Moore EE, Jurkovich GJ, et al: Severe hepatic trauma: a multi-center experience with 1,335 liver injuries. J Trauma 1988; 28:1433-1438.

27 Kudsk KA, Sheldon GF, Lim RC Jr: Atriocaval shunting (ACS) after trauma. J Trauma 1982; 22:81-85.

28 Burch JM, Feliciano DV, Mattox KL: The ateriocaval shunt. Facts and fiction. Ann Surg 1988;207:555-568.

29 Nicol AJ, Hommes M, Primrose R, et al: Packing for control of hemorrhage in major liver trauma. World J Surg 2007;31:569-574.

-30 Alllard MA, Dondero F, Sommacale D, et al: Liver packing during elective surgery: An option that can be considered. World J Surg 2011;35:2493-2498. 